\title{
Estilos de aprendizaje en estudiantes de ciencias de la salud y universidad intercultural de la universidad veracruzana
}

Mtro. José de Jesús Daniel López Muñoz jojedanlm@hotmail.com jeslopez@uv.mx ORCID: 0000-0001-8052-3458

Profesor de Tiempo Completo.

Facultad de Bioanálisis, campus Xalapa, UV

Dra. María Elena Columba Meza Zamora meze53@hotmail.com emeza@uv.mx ORCID: 0000-0002-6306-6176

Profesor de Tiempo Completo. Facultad de Enfermería, campus Xalapa, UV

Dra. Cristina H. Saavedra Vélez cindyenfe@hotmail.com csaavedra@uv.mx ORCID: 0000-0002-9639-8599

Profesor de Tiempo Completo. Facultad de Enfermería, campus Xalapa, UV

Dr. Lauro Fernández Cañedo laferca@hotmail.com lafernandez@uv.mx ORCID:0000-0003-2759-9060 Profesor Tiempo Completo Facultad de Medicina, campus Xalapa, UV

Dr.Omar Lagunes Merino olagunes@uv.mx omarlagunes@hotmail.com ORCID: 0000-0002-2933-4544 Profesor de Tiempo Completo. Facultad de Bioanálisis, campus Xalapa, UV.

Mtro. Crescencio Hernández Osorio cosorio3000@hotmail.com osorio@uv.mx ORCID: 0000-0003-3470-1609 Profesor Técnico Académico de Tiempo Completo.

Facultad de Medicina campus Xalapa

Mtra. Claudia Belén Ortega Planell cbelen1@hotmail.com cbelen1@hotmail.com ORCID: 0000-0003-4434-8810 Profesor Técnico Académico de Tiempo Completo. Facultad de Bioanálisis campus Xalapa, UV Universidad Veracruzana-Xalapa, Veracruz, México 


\section{RESUMEN}

Introducción: Durante años, se ha analizado el proceso del aprendizaje. Los resultados, generan diferentes opiniones de su origen, teorías y principios de cómo el cerebro obtiene el conocimiento y genera diversas opciones para almacenarlo y clasificarlo. Generándose así, el término "Estilo de aprendizaje", refiriendóse al hecho de que cada persona, al aprender, utiliza su método o estrategia.

Objetivo: Determinar el ó los estilos de aprendizaje en estudiantes de licenciaturas de Ciencias de la Salud, región Xalapa y Universidad Veracruzana Intercultural (UVI). Metodología: Estudio cuantitativo, descriptivo, transversal y comparativo. Población y muestra: Alumnos inscritos en el período agosto 2019-enero 2020. La muestra fue probabilística estratificada por licenciatura, con una confiabilidad de 95\%. Instrumento: Honey-Alonso de Estilos de Aprendizaje (CHAEA) en línea, consta de 80 ítems dicotómicos, distribuidos aleatoriamente, evalúa cuatro estilos de aprendizaje (activo, reflexivo, teórico y pragmático). Resultados: Los estilos son similares en enfermería, medicina, psicología y UVI con $50 \%$ y $52 \%$ en el estilo reflexivo, para odontología y bioanálisis $48.1 \%$ y $45.6 \%$ respectivamente, asimismo para nutrición es el $31.4 \%$.

Conclusión: El estilo de aprendizaje encontrado en los estudiantes de ciencias de salud y UVI es el reflexivo, aunque se observaron diversas combinaciones e inclusive individuos con hasta cuatro estilos.

Palabras claves. estilos de aprendizaje; estudiantes; activo; reflexivo; teórico; pragmático. 


\title{
Learning styles in undergraduate students of health sciences and intercultural university from universidad veracruzana
}

\begin{abstract}
Introduction: For years, the learning process has been analyzed. The results generate different opinions of its origin, theories and principles of how the brain obtains knowledge and generates various options to store and classify it. Thus generating the term "Learning Style", referring to the fact that each person, when learning, uses their method or strategy. Objective: To determine the learning style (s) in undergraduate students of Health Sciences, Xalapa region and Intercultural Universidad Veracruzana (UVI). Methodology: Quantitative, descriptive, cross-sectional and comparative study. Population and sample: Students enrolled in the period August 2019-January 2020. The sample was probabilistic stratified by degree, with a reliability of $95 \%$. Instrument: Honey-Alonso of Learning Styles (CHAEA) online, consists of 80 dichotomous items, randomly distributed, assesses four learning styles (active, reflective, theoretical and pragmatic). Results: The styles are similar in nursing, medicine, psychology and ICU with 50\% and 52\% in the reflective style, for dentistry and bioanalysis $48.1 \%$ and $45.6 \%$ respectively, also for nutrition it is $31.4 \%$. Conclusion:The learning style found in health sciences and ICU students is reflective, although various combinations were observed and even individuals with up to four styles.
\end{abstract}

Keywords. learning styles; students; active; reflective; theoretical; pragmatic.

Artículo recibido: 10. Junio. 2021 Aceptado para publicación: 16. Julio. 2021 Correspondencia: jojedanlm@hotmail.com Conflictos de Interés: Ninguna que declarar 


\section{INTRODUCCION}

Estas dos últimas décadas del siglo XX e inicio del presente siglo XXI se ha caracterizado por una serie de transformaciones en los sistemas educativos de cada región, ello como resultado de cambios sociales, económicos, culturales y tecnológicos en nuestra vida académica, lo cual plantea nuevas exigencias y obliga a los sistemas educativos, a una renovación e innovación constante asi como los cambios metodológicos en la educación superior, todo ello para dar respuesta a las demandas y necesidades de los estudiantes y de la sociedad.

$\mathrm{Y}$ es entonces que, a lo largo de estos años, científicos e investigadores se han dado a la tarea de analizar el proceso de aprendizaje en las personas. Son muchos los resultados y los debates que estos han generado, debido a las diferentes opiniones que se tiene acerca de cómo se origina el aprendizaje, sus diferentes teorías y principios, cómo es que nuestro cerebro funciona para obtener el conocimiento y generar diversas opciones para almacenar y clasificar estos.

Esto ha llevado a asegurar, que las personas tanto niños como adultos, aprenden de forma distinta, resulta evidente, toda vez que en el aula conviven una diversidad de estudiantes con características y condiciones diferentes.

A partir de que inician su proceso formativo hay quienes aprenden más rápido que otros, algunos más, presentan dificultades de aprendizaje o atención, incluso aquellos estudiantes que provienen de culturas diversas o se encuentran en entornos o contextos sociales, económicos y culturales diferentes.

Muchos estudiosos del tema enseñanza-aprendizaje, han elaborado métodos que no se pueden generalizar el mismo aprendizaje para cualquier tipo de población y de personas, ya que se siguen enfatizando las diferencias individuales.

Los conocimientos se perciben y se adquieren de manera distinta y además cada individuo tiene preferencia hacia determinadas estrategias cognitivas, que son las que finalmente le ayudarán a dar significado a la nueva información.

En definitiva, la experiencia académica ha demostrado que se tienen diferentes estilos de aprender, toda vez que, el sistema educativo tiene que ver necesariamente con la forma de procesar la información y como los docentes hacen la aplicación del propio conocimiento a 
través de dos opciones: utilizándola como una riqueza potencial o ignorándola y se sigue trabajando de forma tradicional, perdiendo así la oportunidad de lograr un aprendizaje significativo en todos los estudiantes.

Es cierto que en nuestro país se tiende hacia la masificación en la educación pública, los grupos generalmente suelen ser numerosos y las diferencias en un aula constituye hoy día, una realidad cotidiana, por lo que se hace complicado el trabajo académico, siendo un reto el atender las diferencias individuales de los estudiantes.

Diferencias que es necesario analizar a la luz de las nuevas corrientes cognitivas, que ayuden a entender el proceso de la adquisición del conocimiento.

Resulta un tema importante en el mundo de la vida universitaria, el conocer también, cómo aprenden los estudiantes de la intercultural ya que el aprender aprender se ha convertido en una de las capacidades de supervivencia social y que demanda un gran reto para este tipo de estudiantes de la educación.

Todo ello ha llevado a la posibilidad de investigar un aspecto muy concreto de la problemática del aprendizaje denominado "estilos de aprendizaje". Toda vez que existen peculiaridades y modalidades personales para aprender.

Por otro lado se espera que como Institución Universitaria, se enfoque mejor el planteamiento de los entornos y ambientes de aprendizaje para fomentar la innovación educativa, tomando como base el aprendizaje colaborativo en las aulas para aprovechar al máximo los diferentes estilos y precisando en el hecho de que ningún estilo de aprendizaje es permanente y que de acuerdo a las circunstancias puede cambiar, y que además se puede enseñar, lo que los convierte en una fuente de aprendizaje constante.

Es a partir del siglo XX que se promueve tanto en Europa como en Latinoamérica un cambio de orientación curricular en la formación universitaria, destacando Delors, J. (1996) en su informe a la Organización de las Naciones Unidas para la Educación, la Ciencia y la Cultura( UNESCO) en donde explica que la educación a lo largo de la vida se basa en cuatro pilares "el aprender a ser, el aprender hacer, el aprender a conocer y el aprender a vivir juntos" como ejes relevantes para la mejora de los sistemas educativos con un enfoque diferente. 
Por otro lado, se propone una formación basada en competencias, donde el desarrollo de enseñanza -aprendizaje este asentado en el aprender-aprender, orientando la movilización de los cuatro saberes fundamentales a los que hace mención Delors, este desarrollo de competencias exige cambios en las estrategias pedagógicas y en el enfoque curricular de las instituciones de nivel superior siendo el papel central el estudiante

De ahí que, a la competencia se le describe como la capacidad para actuar con eficiencia y satisfacción sobre algún aspecto de la realidad personal, social, natural o simbólica. Siendo que cada competencia viene a ser un aprendizaje complejo que integra habilidades, actitudes y conocimientos y que estará ligado al estilo de aprendizaje del estudiante. (Dovala, 2013)

De manera que la incorporación de los estudiantes a la educación superior e intercultural le enfrenta a una serie de cambios drásticos, toda vez que egresa de un sistema donde el educador dota de todo a los estudiantes y lo mantiene bajo la tutela de algún responsable, por lo tanto, al estar en un ámbito universitario, se tiene que adaptar a un sistema que promueve la independización y adquisición de habilidades.

Ante este hecho, se espera que hayan "alcanzado y desarrollado unos hábitos de estudio, formalizado e instaurado un estilo de aprendizaje, fruto de unas estrategias, que le deben conducir al éxito académico" pero esto no es lo que siempre sucede en el ámbito universitario, se identifican estudiantes con bajos repertorios académicos que no les permiten dar respuesta a las demandas del nuevo proceso de aprendizaje. El bajo desempeño académico que presentan algunos estudiantes cuando ingresan a la educación superior se asocia en gran medida a las estrategias y formas que utilizan para aprender los estudiantes, las cuales no se articulan con las formas de enseñar de los docentes de educación superior. (Valencia, 2014)

Es posible que un gran número de estudiantes universitarios y de la interculturalidad, tengan uno o varios estilos de aprendizaje que no están explorados y explotados, y que solo conozcan las estrategias que les ayudan a comprender los conocimientos que van recibiendo día a día, más no conocen el estilo de aprendizaje que utilizan o que tienen mejor desarrollado para que lo exploten, de ahí que se encuentren limitados al momento de mejorar su aprendizaje. 
Es por lo que, los contenidos impregnados de realidad le permiten al estudiante visualizar un contexto multidisciplinario e interdisciplinario, que dan significado, significancia, relevancia y profundidad, para desarrollarse en lo personal, emocional, social y profesional. De tal forma que, es necesario que tanto el estudiante como el docente, identifiquen y conozcan los estilos de aprendizaje presentes en los estudiantes de ciencias de la salud, región Xalapa y de la Universidad Veracruzana Intercultural de manera que, con ello se pueda generar estrategias que mejoren la comprensión de lo aprendido impactando en el proceso formativo.

\section{OBJETIVOS}

\section{General}

- Determinar el o los estilos de aprendizaje entre los estudiantes de las licenciaturas de Ciencias de la Salud, Químico Clínico, Odontología, Nutrición, Enfermería, Medicina y Psicología región Xalapa y de la Universidad Veracruzana Intercultural.

\section{Específicos}

- Caracterizar sociodemográficamente a la población de estudiantes de Ciencias de la Salud región Xalapa y de la Universidad Veracruzana Intercultural.

- Identificar estilos de aprendizaje por licenciatura, edad y sexo en estudiantes de Ciencias de la Salud región Xalapa y de la Universidad Intercultural, mediante el cuestionario Honey-Alonso de Estilos de Aprendizaje (CHAEA).

- Establecer análisis comparativo de estilos de aprendizaje presentes en estudiantes de ciencias de la salud región Xalapa y de la UVI.

- Difundir los resultados encontrados tanto en las carreras de ciencias de la salud como UVI

\section{MARCO CONCEPTUAL}

El estudio de los estilos de aprendizaje en los estudiantes, responde a la necesidad de la innovación, no como una necesidad personal o docente, sino con un trasfondo pedagógico que pueda actualizar el beneficio integral de los estudiantes.

El concepto de aprendizaje se puede entender como un conjunto de experiencias, las cuales se sintetizan en la vida diaria y que de algún modo cambian algo del individuo. El 
aprendizaje como tal, es un proceso, es decir, forma parte de una serie de eventos o pasos para obtener un conocimiento, un comportamiento e incluso una habilidad nueva.

En el proceso de aprendizaje y conforme una persona va pasando por los diversos estadios de la vida, el proceso cognitivo es adaptable a las necesidades y estímulos que recibe de su entorno. Es durante éste que se forman ciertos patrones que van siendo funcionales al momento de recibir información. Es allí que se van formando modelos por el que toda información será sometida para llegar a la comprensión.

Se ha estudiado el aprendizaje desde el punto de vista de la enseñanza y también la significación del juego y del aprendizaje transferido, así como los tipos de aprendizaje. Los estudiosos del mismo lo han hecho con el objeto de mejorar los métodos de enseñanza, pero no se puede concluir todavía para generalizar el mismo para cualquier tipo de población y de personas ya que se siguen enfatizando las diferencias individuales

Las personas perciben y adquieren los conocimientos de manera distinta y además tienen preferencias hacia determinadas estrategias cognitivas que son las que finalmente les ayudaran a dar significado a la nueva información.

Es importante detenernos a revisar sobre lo que se ha investigado en los últimos años sobre Estilos de aprendizaje (EA), que los estudiantes universitarios tienen como estrategias. Se relacionan con las formas de recopilar, interpretar, organizar y pensar sobre su nueva información.

EA son definidos como operaciones cognitivas básicas, que se integran a patrones de personalidad y que permiten la interacción del sujeto con el entorno, es una forma de interacción sociocognitivos (Luengo y González 2005). Son procedimientos generales de aprendizaje, articulados a lo cognitivo, afectivo y conductual.

EA como características psicológicas, cognitivas, afectivas y fisiológicas presentes en una persona cuando se encuentran en una situación educativa, se relacionan con los modos en que los estudiantes organizan contenidos, construyen conceptos, procesan información y resuelven los problemas. También, se relacionan con las motivaciones y afectos presentes en los momentos de aprendizaje. (López, 2011)

La taxonomía propuesta por Honey y colaboradores (1994), con importantes similitudes a la de García Cué (2006), define los EA como "los rasgos cognitivos, afectivos, 
fisiológicos, de preferencias por el uso de los sentidos, ambiente, cultura, psicología, comodidad, desarrollo y personalidad que sirven como indicadores relativamente estables, de cómo las personas perciben, interrelacionan y responden a sus ambientes de aprendizaje y a sus propios métodos o estrategias en su forma de aprender”. (Garza, Salazar y Olague 2016)

Para esta investigación se consideró la clasificación realizada de acuerdo a las características definidas por Honey y Alonso (1994). Las personas con predominancia en cualquiera de los estilos de aprendizaje pueden definirse de la siguiente manera:

- Activos: Gustan de nuevas experiencias. Son de mente abierta, no escépticos y les agrada emprender nuevas tareas. Son personas que viven en el aquí y el ahora, animador, improvisador, descubridor, arriesgado, espontáneo.

- Reflexivos: Gustan observar las experiencias desde diferentes perspectivas. Reúnen datos para analizarlos con detenimiento antes de llegar a alguna conclusión. Prefieren ser prudentes y mirar bien antes de actuar, ponderado, concienzudo, receptivo, analítico, exhaustivo.

- Teóricos: Suelen ser perfeccionistas. Por lo general, buscan integrar los hechos en teorías coherentes. Gustan de analizar y sintetizar. Para ellos, la racionalidad y la objetividad son aspectos prioritarios, metódico, lógico, objetivo, crítico, estructurado.

- Pragmáticos: Su principal característica se relaciona con la aplicación práctica de las ideas. Son realistas cuando se trata de tomar una decisión o resolver un problema. $\mathrm{Su}$ filosofía es: si funciona, es bueno. Experimentador, práctico, directo, eficaz, realista.

De las aportaciones de Alonso (1995), señalan que a pesar de que no se dan personas con estilos puros, sino perfiles de estilos de aprendizaje, aquellos con los predominios activo, reflexivo, teórico y pragmático poseen varias de las características principales que a continuación se describen: estilo predominante activo la pregunta a la que responden en el aprendizaje es ¿cómo? Lo mejor para ellos es lanzar una actividad que les presente un desafío. Realizar actividades cortas o de resultado inmediato. Cuando hay emoción, drama, crisis. Lo peor es tener un papel pasivo, analizar o interpretar trabajos solos.

Cuando el estilo predominante es el reflexivo, la pregunta a la que responden en el aprendizaje es ¿Por qué? Lo mejor, es cuando pueden ofrecer observaciones y aprendizaje 
es ¿Por qué? Lo mejor, es analizar la situación. Cuando pueden pensar antes de actuar. Lo peor cuando se les obliga a ser el centro de atención. Cuando se les apresura de pasar de una actividad a otra. Cuando tienen que actuar sin planificar.

Cuando el estilo de aprendizaje predominante es teórico la pregunta que se hacen es ¿Qué?. Lo mejor partir de modelos, teorías, sistemas y conceptos que representan un desafío. Cuando tienen la oportunidad de preguntar o indagar. Lo peor las actividades que implican ambigüedad. En situaciones que enfaticen las

De acuerdo con Ordoñez Muñoz et al 2003 en su investigación análisis de los estilos de aprendizaje predominantes entre los estudiantes de ciencias de la salud de la Universidad de Cádiz, Colombia, encontró que el estilo reflexivo corresponde el mayor porcentaje de alumnos analizados, seguido por el pragmático, teórico y activo, además observaron que no existían diferencias estadísticamente significativas entre los alumnos de los primeros tres cursos de formación.

Esta investigación tiene la intención de resaltar en la institución de educación superior denominada Universidad Veracruzana, el centrar su atención en las formas de aprender de los estudiantes y las dificultades que estos tienen asociadas a la adaptación de las nuevas exigencias del contexto universitario. Llevándolo a todo un reto para los docentes, quienes deben tener conocimientos de cómo aprenden sus estudiantes, cuáles son sus conocimientos previos, sus necesidades, sus ritmos, sus motivaciones, expectativas y sus competencias.

\section{METODOLOGÍA}

\section{Perspectiva metodológica y tipo de investigación}

La perspectiva metodológica será cuantitativa, descriptiva, transversal y comparativa.

\section{Fases de abordaje y técnicas}

a) Identificación de la población de estudio y determinación de la muestra.

b) Aplicación de cuestionario Honey- Alonso de estilos de aprendizaje (CHAEA)

c) Análisis de resultados

d) Reporte de resultados

\section{Población y muestra}

Población. Estudiantes inscritos en el período agosto 2019-septiembre 2020 del área de ciencias de la salud, región Xalapa y Universidad Veracruzana Intercultural. 


\section{Muestra.}

Diseño. Se aplicó una muestra probabilística. Para la selección de estudiantes de forma aleatoria simple en cada generación, dentro de cada licenciatura.

Tamaño: Se calculó el tamaño de muestra utilizando la formula general con los siguientes criterios: confiabilidad de $95 \%$, error del $5 \%$ y $\mathrm{p}=0.5$. Una vez que se obtuvo el tamaño de muestra mínima se hizo la asignación proporcional al tamaño de cada entidad académica, obteniendo así un tamaño de muestra mínima de 356 estudiantes.

\section{Instrumento de medición.}

Se aplicó el cuestionario Honey Alonso de EA de manera digital, ya validado por el autor y para este estudio se obtuvo un valor de alfa de cronbach 0.75 , consta de 80 ítems dicotómicos, dicho instrumento clasifica en: Activo, Reflexivo, Teórico y Pragmático.

Se invitó a los estudiantes seleccionados para responder en una computadora o en un dispositivo móvil la version digital del CHAEA.

\section{Criterios de selección}

\section{Inclusión}

- Estudiantes inscritos que cursen las licenciaturas de ciencias de la salud región Xalapa y en la Universidad Intercultural en el período agosto 2019-septiembre 2020.

- Estudiantes inscritos que contesten en su totalidad el cuestionario Honey- Alonso de estilos de aprendizaje (CHAEA) en el período agosto 2019-septiembre 2020.

\section{Exclusión}

- Estudiantes inscritos que no respondan el cuestionario Honey- Alonso de estilos de aprendizaje (CHAEA) en el periodo agosto 2019- septiembre 2020.

- Estudiantes inscritos de otras licenciatura que cursen experiencias educativas en el área Ciencias de la Salud y de la Universidad Veracruzana Intercultural en el periodo agosto 2019-septiembre 2020.

\section{Eliminación}

- Estudiantes inscritos que no contesten completo el cuestionario Honey- Alonso de estilos de aprendizaje (CHAEA) en el momento de su aplicación en el período agosto 2019-septiembre 2020. 


\section{Análisis de datos}

Una vez aplicado el instrumento, la información fue capturada en el programa estadístico SPSS, versión 26 y se hizo una validación de la base datos para minimizar los errores de cálculo

\section{RESULTADOS.}

Figura 1. Distribución de la población por facultad.

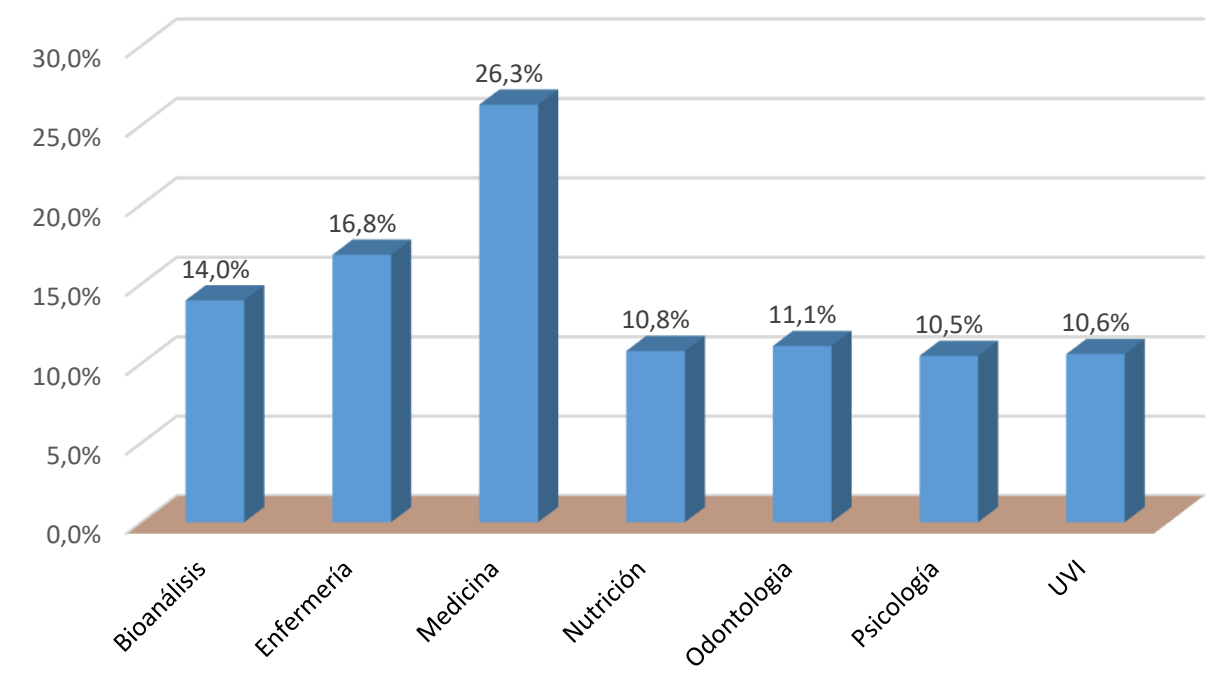

Total: 974 estudiantes.

Figura 1, se observa que el mayor porcentaje de estudiantes que participaron en el estudio son de la facultad de medicina, seguido de la facultad de enfermería y las demás facultades en menor proporción. En cada facultad se cumplió con el tamaño de muestra mínima requerida para este estudio.

Figura 2. Sexo

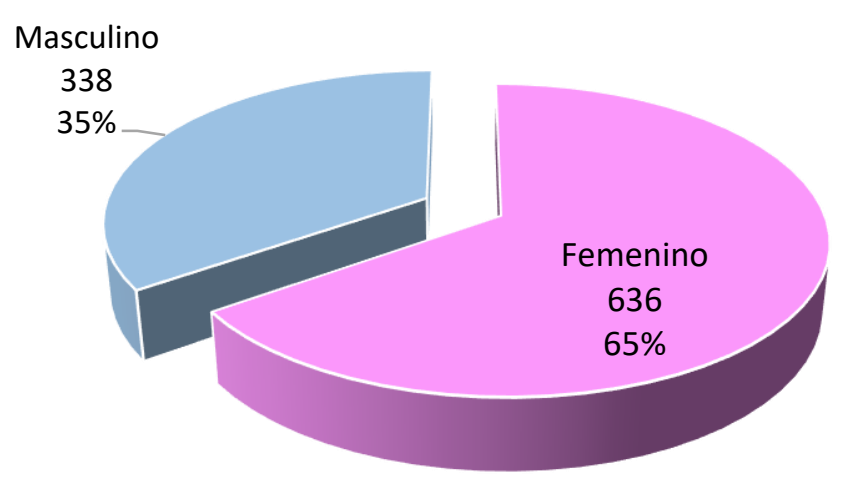


El $65 \%$ de los estudiantes estudiados son del sexo femenino y, el 35\% son masculinos. La edad promedio de los estudiantes evaluados es de 20.1 años, con una desviación estándar de 2.6, la mínima fue de 17 años y la máxima de 41 años.

Figura 3. Edad por facultad

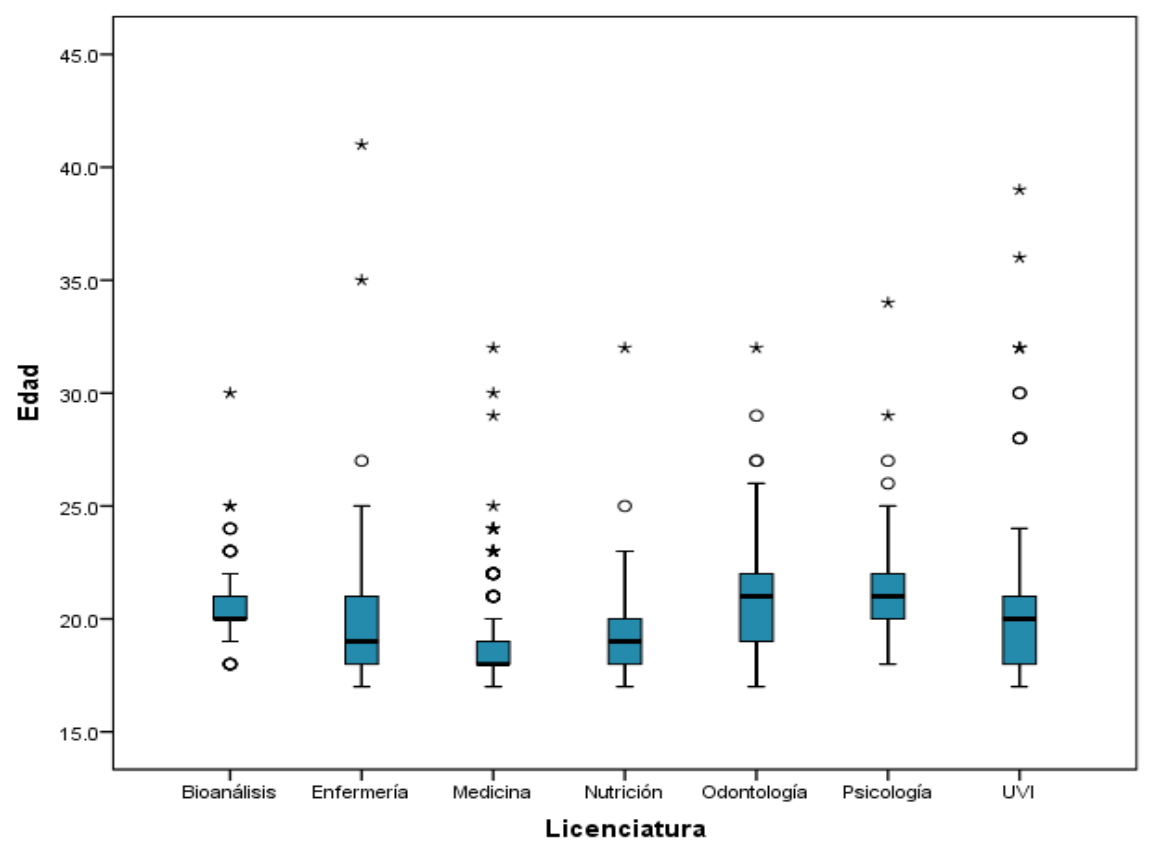

Figura 3, se observa que la mayoría los estudiantes tienen una edad menor de 25 años. La Facultad de enfermería y la UVI es donde se presentan estudiantes de mayor edad.

Figura 4. Autoadscripción Indígena

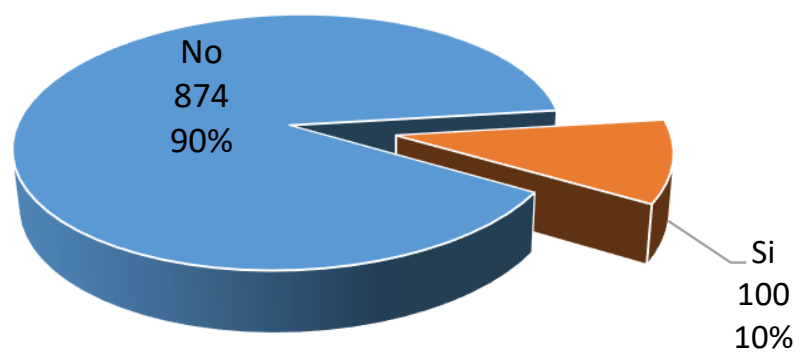

Figura 4, el $10 \%$ de los estudiantes que formaron parte del estudio se autoadscriben como indígenas y el resto como no indígenas. 
Figura 5. Lengua indígena

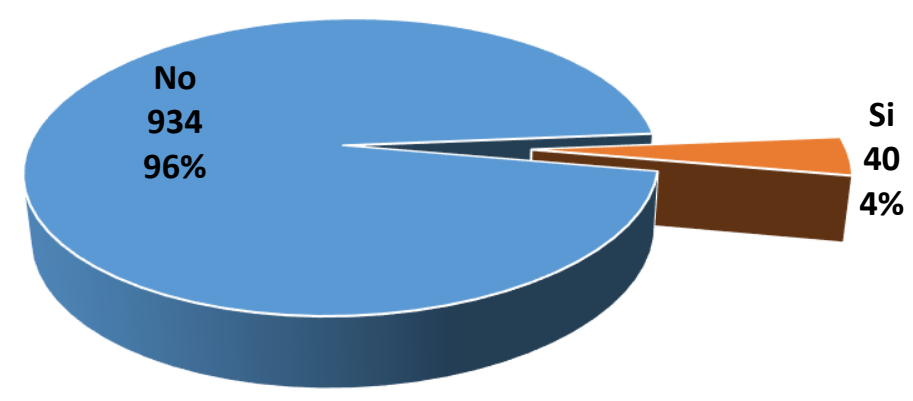

Figura 5, solamente el 4\% (40) de los estudiantes refirió hablar alguna lengua indígena, mientras que el resto refirierón no hacerlo.

Figura 6. Lenguas indígenas

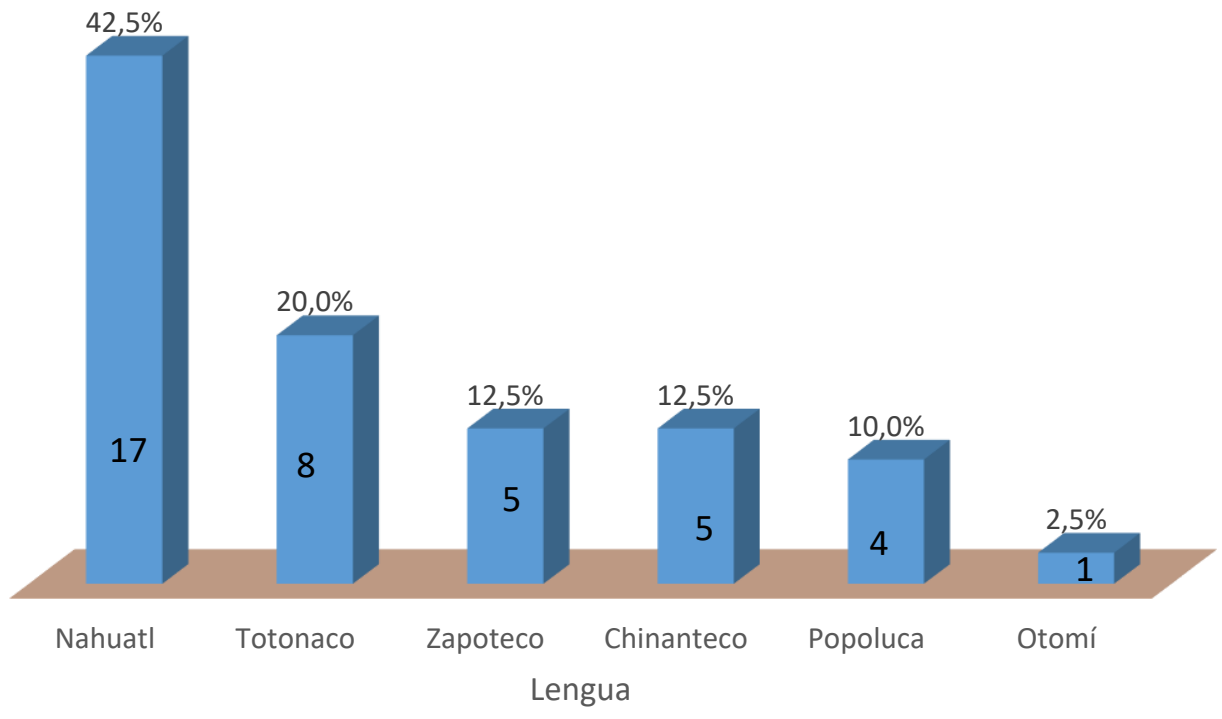

Figura 6, de los hablantes de lenguas indigenas el $42.5 \%$ refiere hablar la lengua náhuatl, el $20 \%$ totonaca, y el resto otras lenguas. 
Figura 7. Estilos de aprendizaje

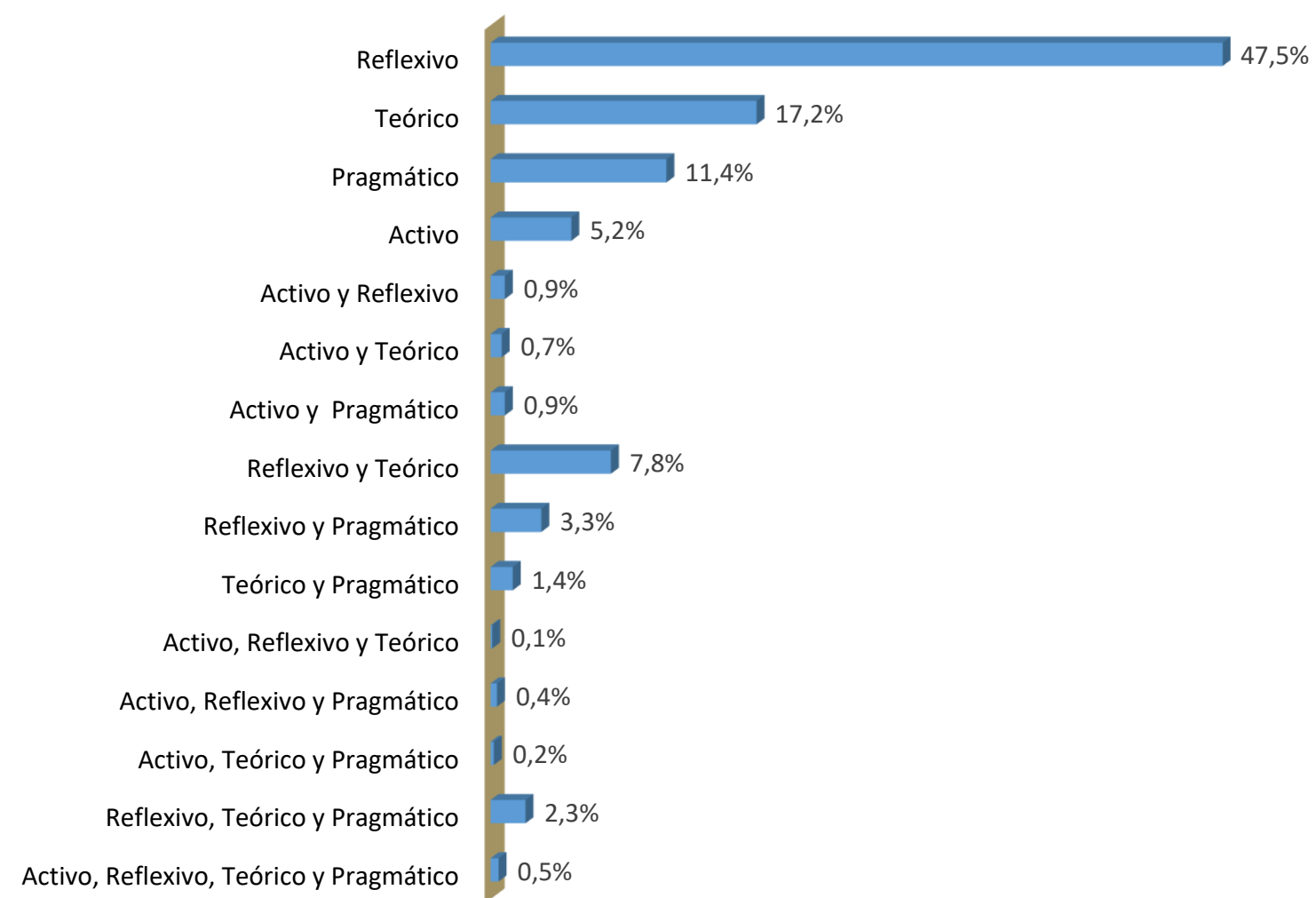

Figura 7, de la población estudiada, el $47.5 \%$ de los estudiantes tienen el estilo de aprendizaje reflexivo, $17.2 \%$ son teóricos, $11.4 \%$ pragmáticos y $5.2 \%$ activos. Se identifica también aquellos con más de un estilo de aprendizaje, $7.8 \%$ tienen estilo de aprendizaje reflexivo y teórico y el resto en menores porcentaje.

Cuadro 1. Estilos de aprendizaje por entidad académica.

\begin{tabular}{|c|c|c|c|c|c|c|c|c|c|c|c|c|c|c|}
\hline \multirow{2}{*}{$\begin{array}{c}\text { Estilo de } \\
\text { aprendizaje }\end{array}$} & \multicolumn{2}{|c|}{ Bioanálisis } & \multicolumn{2}{|c|}{ Enfermería } & \multicolumn{2}{|c|}{ Medicina } & \multicolumn{2}{|c|}{ Nutrición } & \multicolumn{2}{|c|}{ Odontologia } & \multicolumn{2}{|c|}{ Psicología } & \multicolumn{2}{|c|}{ UVI } \\
\hline & $f i$ & $\%$ & fi & $\%$ & $f i$ & $\%$ & fi & $\%$ & $f i$ & $\%$ & fi & $\%$ & $f i$ & $\%$ \\
\hline Reflexivo & 62 & 45.6 & 83 & 50.6 & 128 & 50.0 & 33 & 31.4 & 52 & 48.1 & 53 & 52.0 & 52 & 50.5 \\
\hline Teórico & 21 & 15.4 & 24 & 14.6 & 48 & 18.8 & 21 & 20.0 & 22 & 20.4 & 16 & 15.7 & 16 & 15.5 \\
\hline Pragmático & 20 & 114 & 18 & 11.0 & 27 & 10.5 & 18 & 17.1 & 14 & 13.0 & 8 & 7.8 & 6 & 5.8 \\
\hline Activo & 7 & 5.1 & 13 & & 8 & 3.1 & 8 & 7.6 & 4 & 3.7 & 6 & 5.9 & 5 & 4.9 \\
\hline $\begin{array}{c}\text { Reflexivo y } \\
\text { Teórico }\end{array}$ & 10 & 7.4 & 11 & 6.7 & 24 & 9.4 & 8 & 7.6 & 5 & 4.6 & 6 & 5.9 & 12 & 11.7 \\
\hline
\end{tabular}




\begin{tabular}{|c|c|c|c|c|c|c|c|c|c|c|c|c|c|c|}
\hline $\begin{array}{l}\text { Reflexivo y } \\
\text { Pragmático }\end{array}$ & 5 & 3.7 & 3 & 1.8 & 6 & 2.3 & 4 & 3.8 & 3 & 2.8 & 9 & 8.8 & 2 & 1.9 \\
\hline $\begin{array}{c}\text { Activo y } \\
\text { Pragmático }\end{array}$ & 4 & 2.9 & 1 & 0.6 & 0 & 0.0 & 0 & 0.0 & 0 & 0.0 & 3 & 2.9 & 1 & 1.0 \\
\hline $\begin{array}{c}\text { Teórico y } \\
\text { Pragmático }\end{array}$ & 3 & 2.2 & 1 & 0.6 & 6 & 2.3 & 2 & 1.9 & 0 & 0.0 & 0 & 0.0 & 2 & 1.9 \\
\hline $\begin{array}{l}\text { Activo y } \\
\text { Teórico }\end{array}$ & 0 & 0.0 & 2 & 1.2 & 1 & 0.4 & 4 & 3.8 & 0 & 0.0 & 0 & 0.0 & 0 & 0.0 \\
\hline $\begin{array}{l}\text { Activo y } \\
\text { Reflexivo }\end{array}$ & 1 & 0.7 & 0 & 0.0 & 1 & 0.4 & 1 & 1.0 & 4 & 3.7 & 0 & 0.0 & 2 & 1.9 \\
\hline $\begin{array}{c}\text { Activo, } \\
\text { Reflexivo y } \\
\text { Teórico }\end{array}$ & 1 & 0.7 & 0 & 0.0 & 0 & 0.0 & 0 & 0.0 & 0 & 0.0 & 0 & 0.0 & 0 & 0.0 \\
\hline $\begin{array}{c}\text { Activo, } \\
\text { Reflexivo y } \\
\text { Pragmático }\end{array}$ & 1 & 0.7 & 1 & 0.6 & 0 & 0.0 & 1 & 1.0 & 1 & 0.9 & 0 & 0.0 & 0 & 0.0 \\
\hline $\begin{array}{c}\text { Activo, } \\
\text { Teórico y } \\
\text { Pragmático }\end{array}$ & 0 & 0.0 & 1 & 0.6 & 1 & 0.4 & 0 & 0.0 & 0 & 0.0 & 0 & 0.0 & 0 & 0.0 \\
\hline $\begin{array}{c}\text { Reflexivo, } \\
\text { Teórico y } \\
\text { Pragmático }\end{array}$ & 1 & 0.7 & 5 & 3.0 & 6 & 2.3 & 2 & 1.9 & 3 & 2.8 & 0 & 0.0 & 5 & 4.9 \\
\hline $\begin{array}{c}\text { Activo, } \\
\text { Reflexivo, } \\
\text { Teórico y } \\
\text { Pragmático }\end{array}$ & 0 & 0.0 & 1 & 0.6 & 0 & 0.0 & 3 & 2.9 & 0 & 0.0 & 1 & 1.0 & 0 & 0.0 \\
\hline Total & 136 & 100.0 & 164 & 100.0 & 256 & 100.0 & 105 & 100 & 108 & 100 & 102 & 100.0 & 103 & 100.00 \\
\hline
\end{tabular}

En el cuadro 1, se identificó que los estilos de aprendizaje son muy similares en las entidades estudiadas dentro de las licenciaturas de enfermeria, medicina, psicología y la UVI los porcentajes son similares entre un 50 y 52\% en el estilo reflexivo, odontología y bioanálisis con $48.1 \%$ y $45.6 \%$ respectivamente, siendo la licenciatura de nutrición quien refleja el porcentaje mas bajo de este estilo con $31.4 \%$. 
Cuadro 2. Estilos de aprendizaje de Ciencias de la Salud y la UVI

\begin{tabular}{|c|c|c|c|c|}
\hline \multirow{2}{*}{ Estilos de aprendizaje } & \multicolumn{2}{|c|}{ Ciencias de salud } & \multicolumn{2}{|c|}{ UVI } \\
\hline & fi & $\%$ & fi & $\%$ \\
\hline Reflexivo & 411 & 47.2 & 52 & 50.5 \\
\hline Teórico & 152 & 17.5 & 16 & 15.5 \\
\hline Pragmático & 105 & 12.1 & 6 & 5.8 \\
\hline Activo & 46 & 5.3 & 5 & 4.9 \\
\hline Reflexivo y Teórico & 64 & 7.3 & 12 & 11.7 \\
\hline Reflexivo y Pragmático & 30 & 3.4 & 2 & 1.9 \\
\hline Activo y Pragmático & 8 & 0.9 & 1 & 1.0 \\
\hline Teórico y Pragmático & 12 & 1.4 & 2 & 1.9 \\
\hline Activo y Teórico & 7 & 0.8 & 0 & 0.0 \\
\hline Activo y Reflexivo & 7 & 0.8 & 2 & 1.9 \\
\hline Activo, Reflexivo y Teórico & 1 & 0.1 & 0 & 0.0 \\
\hline Activo, Reflexivo y Pragmático & 4 & 0.5 & 0 & 0.0 \\
\hline Activo, Teórico y Pragmático & 2 & 0.2 & 0 & 0.0 \\
\hline Reflexivo, Teórico y Pragmático & 17 & 2.0 & 5 & 4.9 \\
\hline $\begin{array}{l}\text { Activo, Reflexivo, Teórico } \\
\text { Pragmático }\end{array}$ & $\mathrm{y}$ & 0.6 & 0 & 0.0 \\
\hline Total & 871 & 100.0 & 103 & 100.000 \\
\hline
\end{tabular}

Cuadro 2, comparativamente entre estudiantes de Ciencias de la Salud, región Xalapa y estudiantes de la UVI, el estilo reflexivo predomina en ambos grupos con $47.2 \%$ y $50.5 \%$ respectivamente. Seguido del estilo de aprendizaje teórico con $17.5 \%$ y $15.5 \%$ respectivamente.

Cuadro 3. Estilos de aprendizaje de acuerdo al sexo.

\begin{tabular}{lcccc}
\hline \multirow{2}{*}{ Estilos de aprendizaje } & \multicolumn{2}{c}{ Femenino } & \multicolumn{2}{c}{ Masculino } \\
& $\boldsymbol{f i}$ & $\boldsymbol{\%}$ & $\boldsymbol{f i}$ & $\boldsymbol{\%}$ \\
\hline Reflexivo & 316 & 49.7 & 147 & 43.5 \\
Teórico & 107 & 16.8 & 61 & 18.0 \\
Pragmático & 63 & 9.9 & 48 & 14.2 \\
Activo & 35 & 5.5 & 16 & 4.7 \\
Activo y Reflexivo & 8 & 1.3 & 1 & 0.3 \\
Activo y Teórico & 6 & 0.9 & 1 & 0.3 \\
Activo y Pragmático & 5 & 0.8 & 4 & 1.2 \\
Reflexivo y Teórico & 48 & 7.5 & 28 & 8.3 \\
Reflexivo y Pragmático & 15 & 2.4 & 17 & 5.0
\end{tabular}




$\begin{array}{lcccc}\text { Teórico y Pragmático } & 11 & 1.7 & 3 & 0.9 \\ \text { Activo, Reflexivo y Teórico } & 1 & 0.2 & 0 & 0.0 \\ \text { Activo, Reflexivo y Pragmático } & 4 & 0.6 & 0 & 0.0 \\ \text { Activo, Teórico y Pragmático } & 2 & 0.3 & 0 & 0.0 \\ \text { Reflexivo, Teórico y Pragmático } & 11 & 1.7 & 11 & 3.3 \\ \text { Activo, Reflexivo, Teórico y Pragmático } & 4 & 0.6 & 1 & 0.3 \\ \text { Total } & \mathbf{6 3 6} & \mathbf{1 0 0 . 0} & \mathbf{3 3 8} & \mathbf{1 0 0 . 0}\end{array}$

Cuadro 3, respecto al estilo de aprendizaje en relación al sexo, se encontró que el 49.7\% tienen un estilo de aprendizaje reflexivo y corresponde al sexo femenino; el $43.5 \%$ al masculino. Con respecto al estilo de aprendizaje teórico,16.8\% son del sexo femenino y $18 \%$ del masculino.

Figura 8. Estilos de aprendizaje por edad.

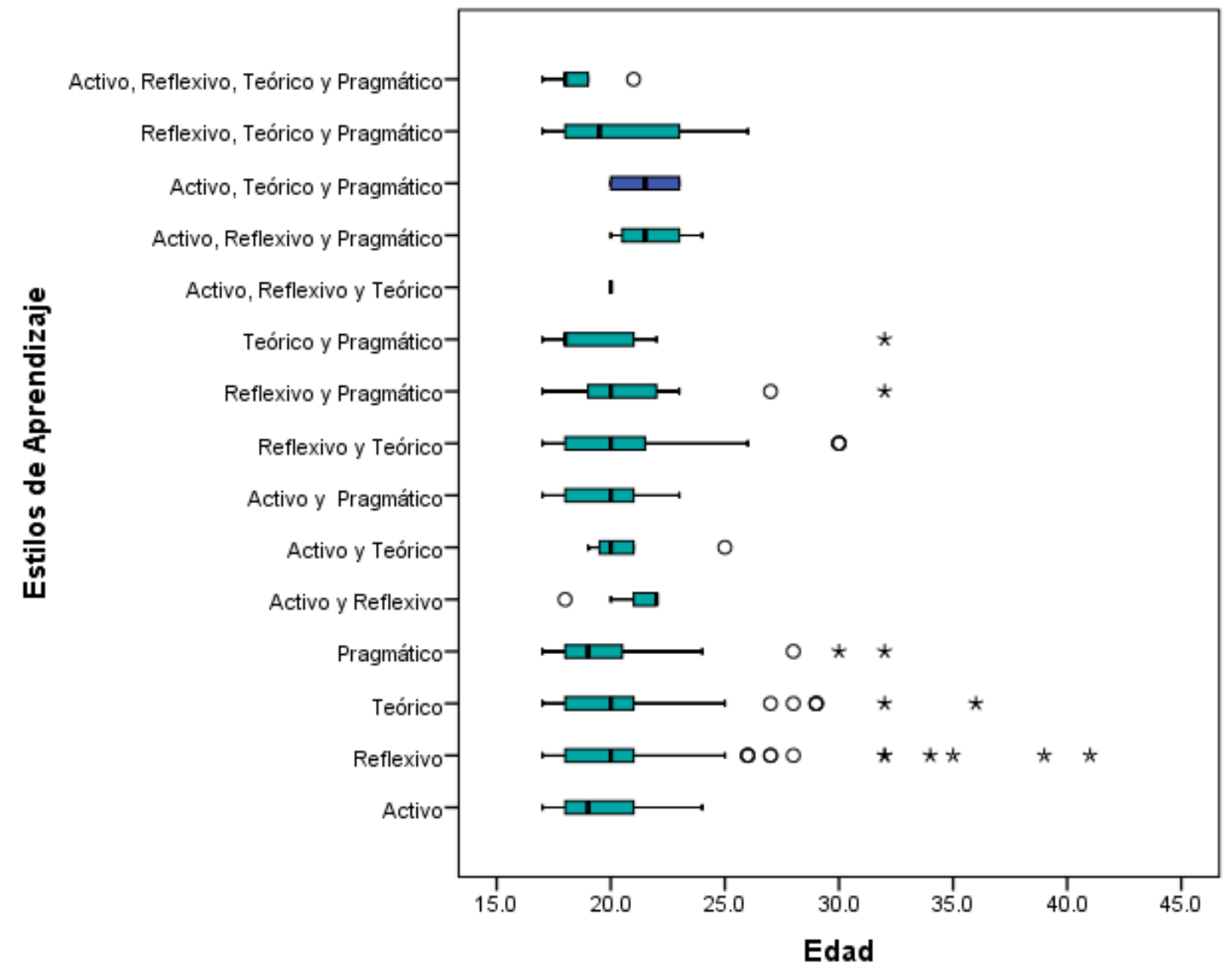

Figura 8, la distribución de la edad de los estudiantes fue similare en todos los estilos de aprendizaje. Los de mayor edad se encuentran como reflexivo y teórico. 


\section{CONCLUSIONES}

El estilo de aprendizaje predominante, al analizar los resultados obtenidos en las licenciaturas de Ciencias de la Salud de la Universidad Veracruzana y la UVI, fue el reflexivo, seguido por el teórico, después el pragmático y por último el activo.

\section{DISCUSION}

En este estudio se encontró que los estilos de aprendizaje en orden de frecuencia fue el reflexivo, teórico, pragmático y el activo, lo que coincide con los resultados obtenidos por Salas (2014) en estudiantes universitarios de movimiento humano y calidad de vida en Costa Rica, cabe señalar que Alonso y colaboradores en 1994 manifiesta que el estilo de aprendizaje reflexivo es caracteristico de los estudiantes de las licenciaturas que conforman las ciencias de la salud.

Asi mismo Bravo y Alonso 2007, Canalejas et al 2005, encontraron que en estudiantes universitarios de ciencias de salud el Estilo de aprendizaje reflexivo es el que prevalece en mayor proporción, ya que este, ademas de ser caracteristico de cada individuo es influenciado por el docente y las estrategias de enseñanza que lo predisponen hacia un estilo reflexivo. Lo que permite considerar que los estudiantes tienden a ser receptivos, analíticos, y prefieren la aplicación de lo aprendido en las aulas de clase, lo que implica una valiosa información para los docentes, ademas se observa la combinación de estilos siendo la reflexivo-teórico la encontrada en mayor proporción. Esta conclusión debe ser considerada al momento de diseñar los métodos de enseñanza por parte de los profesores. Sin embargo, lo reportado por González Garza y colaboradores en 2016, el estilo predominante en estudiantes de enfermeria de la Universidad de Querétaro, es el pragmático y en menor porcentaje el reflexivo, lo que difiere de los resultados reportados en esta investigación.

A través de los resultados se reflexiona la necesidad de que también sean identificados los estilos de enseñanza de los profesores, a fin de promover estrategias didácticas acordes a los estudiantes y favorecer un tránsito escolar y disminuir la deserción escolar también propuesto por Donoso 2005. 


\section{REFERENCIAS BIBLIOGRAFICAS}

Delors, J. (2013). Los cuatro pilares de la educación. Galileo, (23).

Dovala, J. M. C. original: Estrategias de Enseñanza para el Aprendizaje por Competencias.

Valencia, L. I. (2014). Estilos de Aprendizaje: una apuesta por el desempeño académico de los estudiantes en la Educación Superior. Encuentros, 12(2), 25-34.

Luengo, R., \& González, J. J. (2005). Relación entre los estilos de aprendizaje, el rendimiento en matemáticas y la elección de asignaturas optativas en alumnos de enseñanza secundaria obligatoria (ESO). Unión. Revista Iberoamericana de Educación Matemática, 3, 25-46.

López Aguado, M. (2011). Estrategias de aprendizaje en estudiantes universitarios. Diferencias por género, curso y tipo de titulación.

Honey, P., Alonso, C., Domingo, J., \& Domingo, J. (1994). Los estilos de aprendizaje: procedimientos de diagnóstico y mejora. España, Bilbao: Ediciones El Mensajero.

Alonso, C., Gallego,D.J.,\& Honey, P.(1995). Los estilos de aprendizaje: procedimientos de diagnóstico y mejora. Editorial Mensajero.

Cué, J. L. G. (2006). Los Estilos de Aprendizaje y las Tecnologías de la Información y la Comunicación en la Formación del Profesorado (Doctoral dissertation, UNED. Universidad Nacional de Educación a Distancia (España)).

Garza, M. D. R. R., Salazar, M. F. S., \& Olague, R. I. E. (2016). Análisis de los estilos de aprendizaje en estudiantes universitarios del área de la salud. Revista de Estilos de Aprendizaje, 9(17).

Muñoz, F. O., Rosety-Rodríguez, M., \& Rosety-Plaza, M. (2003). Análisis de los estilos de aprendizaje predominantes entre los estudiantes de ciencias de la salud. Enfermería global, 2(2).

Salas-Cabrera, J. (2014). Estilos de aprendizaje en estudiantes de la Escuela de Ciencias del Movimiento Humano y Calidad de Vida, Universidad Nacional, Costa Rica. Revista electronica EDUCARE, 18(3), 159-171.

Bravo Hernández, P. L., \& Alfonso Romero, M. (2007). Comportamiento de actividades que desarrollan estilos de aprendizaje en las guías didácticas de la asignatura Morfofisiología Humana I. Educación Médica Superior, 21(4), 0-0. 
Canalejas Pérez, M., Martínez Martín, M., Pineda Ginés, M., Vera Cortés, M. L., Soto González, M., Martín Marino, Á., \& Cid Galán, M. (2005). Estilos de aprendizaje en los estudiantes de enfermería. Educación médica, 8(2), 33-40.

Donoso, M. M. (2005). El educador como agente reflexivo en el proceso de formación educativa. Horizontes Educacionales, (10), 71-76. 